\title{
Study on gastric cancer blood plasma based on surface-enhanced Raman spectroscopy combined with multivariate analysis
}

\author{
FENG Shang Yuan ${ }^{1}$, PAN JianJi $^{2}$, WU YanAn ${ }^{3}$, LIN Duo $^{1}$, CHEN YanPing ${ }^{2}$, XI GangQin ${ }^{1}$, \\ LIN JuQiang ${ }^{1} \&$ CHEN Rong ${ }^{1 *}$ \\ ${ }^{1}$ Key Laboratory of OptoElectronic Science and Technology for Medicine, Ministry of Education of China, \\ Fujian Normal University, Fuzhou 350007, China; \\ ${ }^{2}$ Fujian Provincial Tumor Hospital, Fuzhou 350001, China; \\ ${ }^{3}$ Fujian Provincial Hospital, Fuzhou 350001, China
}

Received December 6, 2010; accepted May 24, 2011; published online August 2, 2011

\begin{abstract}
A surface-enhanced Raman spectroscopy (SERS) method combined with multivariate analysis was developed for non-invasive gastric cancer detection. SERS measurements were performed on two groups of blood plasma samples: one group from 32 gastric patients and the other group from 33 healthy volunteers. Tentative assignments of the Raman bands in the measured SERS spectra suggest interesting cancer-specific biomolecular changes, including an increase in the relative amounts of nucleic acid, collagen, phospholipids and phenylalanine and a decrease in the percentage of amino acids and saccharide in the blood plasma of gastric cancer patients as compared with those of healthy subjects. Principal components analysis (PCA) and linear discriminant analysis (LDA) were employed to develop effective diagnostic algorithms for classification of SERS spectra between normal and cancer plasma with high sensitivity (79.5\%) and specificity (91\%). A receiver operating characteristic (ROC) curve was employed to assess the accuracy of diagnostic algorithms based on PCA-LDA. The results from this exploratory study demonstrate that SERS plasma analysis combined with PCA-LDA has tremendous potential for the non-invasive detection of gastric cancers.
\end{abstract}

surface-enhanced Raman spectroscopy (SERS), blood plasma, gastric cancer, detection

Citation: Feng S Y, Pan J J, Wu Y A, et al. Study on gastric cancer blood plasma based on surface-enhanced Raman spectroscopy combined with multivariate analysis. Sci China Life Sci, 2011, 54: 828-834, doi: 10.1007/s11427-011-4212-8

Raman spectroscopy (RS) can provide fingerprint type information on the structure and conformation of specific molecular species [1]. It has been used for molecule detection and material identification. RS has also been widely applied for biomedical research [2-8]. Changes in the conformation and components of human tissues and cells can be explored by detecting species such as DNA, protein, and lipid. This technique has attracted considerable attention due to its great potential for cancer diagnosis. For example, several researchers have studied the blood serum and cells

*Corresponding author (email: chenr@fjnu.edu.cn) obtained from gastric cancer patients and healthy volunteers by Raman spectra under a $523 \mathrm{~nm}$ green laser, which was considered effective for gastric cancer screening, prognosis monitoring and efficacy evaluation [9-11]. Tao et al. [12] applied RS to analyze the apoptosis of human gastric cancer cells induced by cisplatin and demonstrated that cisplatin could induce the apoptosis of gastric cancer cells by decreasing the nucleic acid and protein content. Zhang et al. [13], Ling et al. [14] and Tang et al. [15] also used RS to study in vitro tissues and showed a number of differences between normal and gastric cancer tissue, indicating that RS would become an effective assistant method for gastric 
cancer diagnosis.

All studies mentioned above used conventional Raman spectroscopy for gastric cancer detection. However, Raman scattering suffers the disadvantage of extremely poor efficiency, especially for biological molecules, because of its inherently small cross-section [16]. This inefficient scattering requires the use of high laser power and/or long data collection times for spectral acquisition that could damage biological samples. Meanwhile, the Raman spectra of biological samples are often superimposed on a strongly fluorescent background that may be overwhelming and make it difficult to analyze the Raman signals.

Surface-enhanced Raman scattering (SERS) was first reported in 1974 by Fleischman et al. [17], who observed the surface-enhanced Raman spectrum of pyridine adsorbed on electrochemically roughened silver electrodes. With the SERS technique, Raman signals can be enhanced dramatically, with low laser power and little damage to biological molecules when the probed molecules are attached to nano-textured metallic surfaces, while the autofluorescent background can be greatly reduced at the same time. In addition, the SERS technology has a high detection sensitivity and is capable of realizing single molecule detection. Therefore, it is particularly suitable for research into biological molecules [18].

Recently, the SERS technique has been widely used in biomedical applications, including detection of various biological agents (viruses, bacteria, and DNA) [19-21]. Cancer diagnosis is another potential application for the SERS technique. Most current oncology applications are focused on developing a SERS-based immunoassay, which relies on a specific interaction between an antigen and a complementary antibody [22-29].

Gastric cancer is one of the most common malignancies and has the highest incidence among all malignancies in China. Eastern Asia, South American and Eastern Europe have very high incidences of stomach cancers, accounting for 600000 deaths worldwide every year. Human blood plays an important role in our body, can be sampled conveniently and is closely related to the state of human health. Therefore, changes of blood may be associated with the occurrence of some diseases.

Our group has recently applied SERS to blood plasma analysis for nasopharyngeal cancer detection and obtained good preliminary results [30]. In this paper, we explore the use of SERS spectroscopy combined with multivariate analysis for non-invasive gastric cancer detection

\section{Materials and methods}

$\mathrm{AgNO}_{3}$ (mass fraction $\geqslant 99.8 \%$, Reagent No. 1 Factory of Shanghai); $\mathrm{NaOH}$ (Sinopharm Chemical Reagent Co., Ltd.). All reagents used in experiments were of analytical grade.

\subsection{Preparation of silver colloids and human blood plasma samples}

Silver (Ag) colloids were prepared by the deoxidizing method in accordance with the report by Nicolae Leopold [31]. Briefly, a total of $4.5 \mathrm{~mL}$ of sodium hydroxide solution $\left(0.1 \mathrm{~mol} \mathrm{~L}^{-1}\right)$ was added to $5 \mathrm{~mL}$ of $0.06 \mathrm{~mol} \mathrm{~L}^{-1}$ hydroxylamine hydrochloride solution. The mixture was then added rapidly to $90 \mathrm{~mL}$ of $0.0011 \mathrm{~mol} \mathrm{~L}^{-1}$ silver nitrate aqueous solution and the resulting mixture shaken until a homogeneous mixture was obtained. The resulting colloid had a milky gray color. The colloidal particles were characterized by an absorption maximum at $418 \mathrm{~nm}$ with a full width at half-maximum of approximately $100 \mathrm{~nm}$ (Figure 1). The inserted picture in the figure shows a transmission electron microscopy (TEM) photograph of the prepared silver colloid. The particle sizes followed a normal distribution with a mean diameter of $34 \mathrm{~nm}$ and standard deviation of 5 $\mathrm{nm}$. The silver colloidal solution was concentrated by centrifugation at $10000 \mathrm{r} \mathrm{min}^{-1}$ for $10 \mathrm{~min}$, discarding a portion of the supernatant and adjusting the final concentration for use later with the plasma samples.

Two subject groups were studied in this work. The first group consisted of 32 patients with confirmed clinical and histopathological diagnosis of gastric cancers. The second group consisted of 33 healthy volunteers as a control group. After $12 \mathrm{~h}$ of overnight fasting, single $3 \mathrm{~mL}$ peripheral blood samples were obtained from the study subjects between 7:00 and 8:00 a.m., using 10 $\mu \mathrm{L}$ of EDTA-K2 $\left(15 \mathrm{~g} \mathrm{~L}^{-1}\right)$ as anticoagulant. Blood cells were removed by centrifugation at $2000 \mathrm{r} \mathrm{min}^{-1}$ for $15 \mathrm{~min}$ to obtain the blood plasma. Then, $200 \mu \mathrm{L}$ blood plasma was mixed with $200 \mu \mathrm{L}$ silver colloidal nanoparticles in a $1: 1$ proportion. This was mixed with a pipette tip to create as homogeneous a mixture as possible. The mixture was incubated for $2 \mathrm{~h}$ at $4^{\circ} \mathrm{C}$ before measurement.

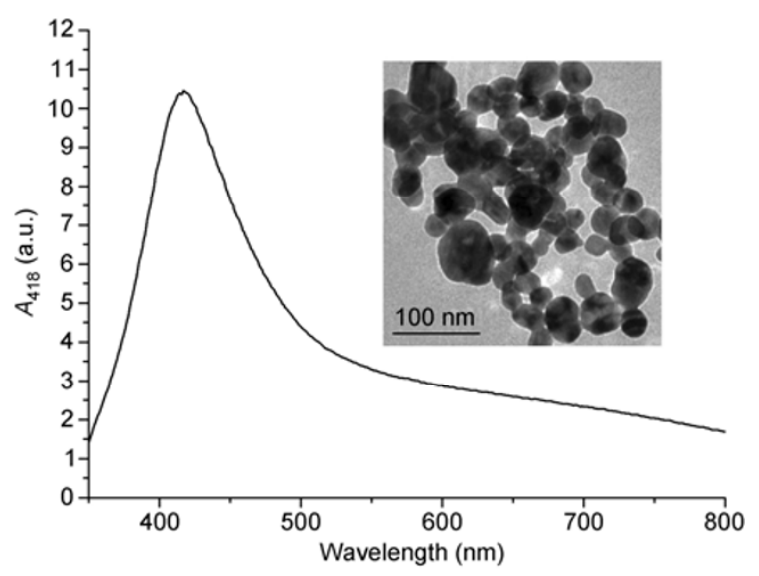

Figure 1 The UV/visible absorption spectrum of silver colloid. The absorption maximum is located at $418 \mathrm{~nm}$. The inserted picture shows a TEM micrograph of silver nanoparticles. 


\subsection{SERS measurements}

A drop of the above mixture was transferred onto a rectangle aluminum plate $(99.99 \%)$ and dried. The SERS spectra were recorded with a confocal Raman micro-spectrometer (Renishaw, Great Britain) in the range of $450-1800 \mathrm{~cm}^{-1}$ under a $785 \mathrm{~nm}$ and $5 \mathrm{~mW}$ diode laser excitation. The spectra were collected in backscattering geometry using a microscope equipped with a Leica $20 \times$ objective with a $10 \mathrm{~s}$ integration time. Three spectra were obtained from a sample in different positions and the mean spectra from those were taken for analysis.

\section{Results and discussion}

The three spectra shown in Figure $2 \mathrm{~A}-\mathrm{C}$ were measured under the same instrumentation set-up. Figure 2A and B shows the SERS spectra of patient blood plasma with added Ag sol and the regular Raman spectrum of the blood plasma without Ag sol, respectively. A comparison of Figure 2A and $\mathrm{B}$ demonstrated that the intensity of many dominant vibrational bands has been increased dramatically by SERS, indicating that there were strong interactions between the silver colloids and the blood plasma. Because of this interaction, biochemical substances in plasma samples can closely adsorb on the silver colloid particle surfaces, thus leading to an extraordinary enhancement in the intensity of the Raman scattering. Only a few Raman peaks could be observed in the native blood plasma without the addition of silver solution because most of the Raman signals were masked by the large autofluorescence background. An obvious decrease in the intensity of the fluorescence background and clearly resolved, high intensity Raman bands were observed in the SERS spectra. Figure 2C shows the background Raman signal of the anticoagulant with added $\mathrm{Ag}$

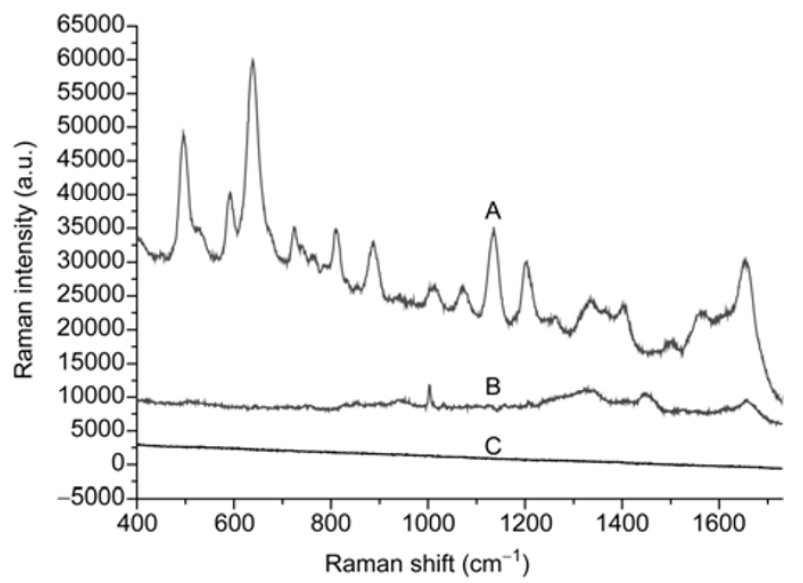

Figure 2 SERS spectrum of a blood plasma sample obtained by mixing the plasma with the Ag colloid at a 1:1 proportion (A), a regular Raman spectrum of the same plasma sample without the silver sol (B), and the background Raman signal of the anticoagulant agent mixed with the $\mathrm{Ag}$ colloid (C). sol. We see no interference signal in the spectral range of interest.

To reduce the spectral intensity variations between different spectra and facilitate more accurate spectral shape analysis, all measured SERS spectra were normalized by the integrated area under the curve in the $450-1730 \mathrm{~cm}^{-1}$ wavenumber range after the removal of fluorescence background from the original SERS data. Figure 3 compares the normalized mean SERS spectra obtained from 33 normal subject blood plasma samples (red line) and 32 gastric cancer patient plasma samples (black line). Shown at the top are the mean SERS spectra for healthy subject blood plasma and gastric cancer patient blood plasma with the standard deviations overlaid as shaded color fill. There are spectral regions where the standard deviations do not overlap and the differences are thus significant and reproducible. It can be seen that while significant SERS spectral differences exist between normal and tumor samples, primary SERS peaks at 494, 589, 638, 725, 813, 886, 1004, 1075, 1134, $1207,1330,1445,1580$ and $1653 \mathrm{~cm}^{-1}$ can all be observed in both normal and tumor blood plasmas, with the strongest signals at 494, 638 and $1134 \mathrm{~cm}^{-1}$. The normalized intensities of SERS peaks at 494, 589, 638, 813, and $1134 \mathrm{~cm}^{-1}$ are more intense for normal plasma than for tumor plasma, while SERS bands at 1330,1445 and $1580 \mathrm{~cm}^{-1}$ are greater in gastric cancer plasma samples. These normalized intensity differences can be viewed more clearly in the difference SERS spectrum between tumor and normal plasma samples (bottom in Figure 3). For example, the shoulder band variation at 1450 and $1580 \mathrm{~cm}^{-1}$ for gastric cancer plasma becomes more obvious on the difference spectrum. These spectral differences are explored in greater detail for blood plasma classification through principal component analysis in the next section.

\subsection{Analysis of the SERS spectra}

To test the capability of silver nanoparticles based plasma SERS spectra for differentiating gastric cancer from normal samples, principal component analysis (PCA) combined with linear discriminate analysis (LDA) was performed on the measured blood plasma SERS spectra. PCA is a statistical technique for simplifying complex data sets and determining the key variables in a multi- dimensional data set that best explain the differences in the observations. First, the fluorescence background of the original SERS data was removed using a fifth-order polynomial fitting algorithm [32], then each spectrum was normalized by the integrated area under the curve. The entire normalized SERS spectrum data set was fed into the SPSS software package (SPSS Inc., Chicago) for PCA-LDA analysis. Independent-sample T test on all the PC scores comparing normal and cancerous groups showed that there were three PCs (PC1, PC2 and PC7) that were most diagnostically significant $(P<<0.05)$ for discriminating normal and cancerous groups. 
Figure 4 shows the result of a three-dimensional scatter plot of PC scores (PC1, PC2 and PC7 as the three axes) for the healthy (black circles) and cancer (red triangles) groups. We can clearly see that they were distributed in separate areas except for a small overlap, which means that we are able to discriminate between the SERS spectra of the cancer group and the healthy control group.

A similar three-dimensional scatter plot of PC scores (PC1, PC2 and PC4 as the three axes) as calculated from the original SERS data for the healthy (black circles) and cancer (red triangles) groups is also presented in Figure 5. In contrast to Figure 4, there is significant overlap between the cancer group and the healthy control group, indicating that the diagnosis effect will became poor without the SERS data pretreatment.

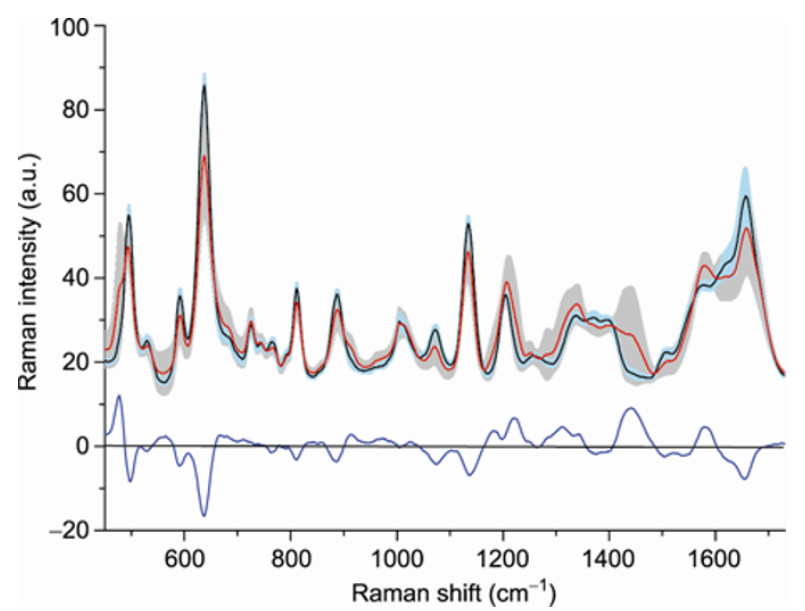

Figure 3 Comparison of the mean spectrum for normal blood plasma (black line, $n=33$ ) versus that of gastric cancer (red line, $n=32$ ). The shaded areas represent the standard deviations of the means. Also shown at the bottom is the difference spectrum.

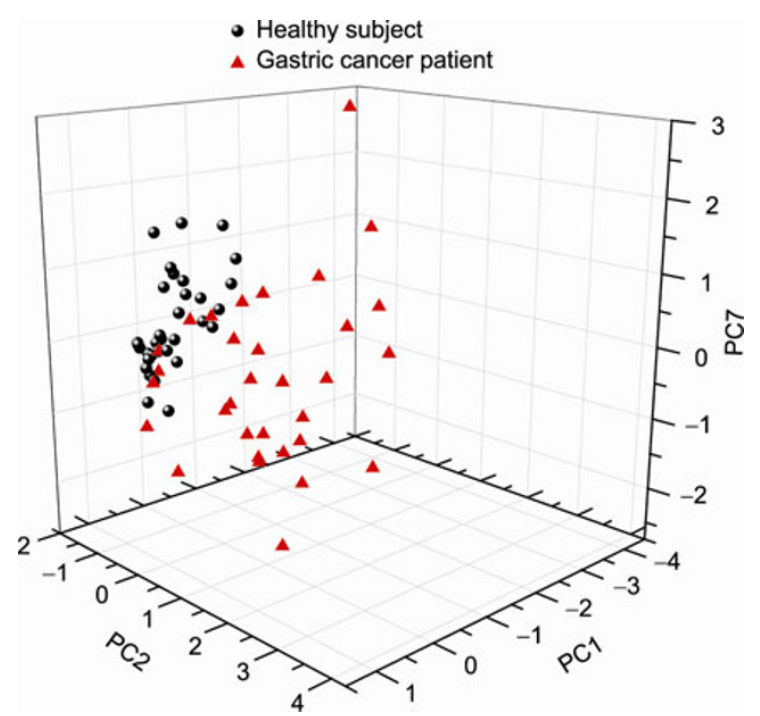

Figure 4 A three-dimensional mapping of the PCA result for the gastric cancer group (red triangles) and the healthy volunteer group (black circles) with PC1, PC2 and PC7 as the three axes.
To actually incorporate all significant spectral features, LDA was used to generate diagnostic algorithms using the PC score for the three most significant PCs (PC1, PC2 and PC7). To prevent over-training, the leave-one-out and crossvalidation procedures were used. Figure 6 shows the posterior probabilities of belonging to the normal (black circles) and cancer (red triangles) in the LDA model. Using a discrimination threshold of 0.5 , the diagnostic sensitivity and specificity for detecting gastric cancer were $79.5 \%$ and $91 \%$, respectively. On the other hand, the PCA-LDA based diagnostic algorithms calculated from the original SERS data yielded a diagnostic sensitivity of $75 \%$ and specificity of $66.7 \%$ for separating cancerous samples from normal samples. After the removal of the fluorescence background and

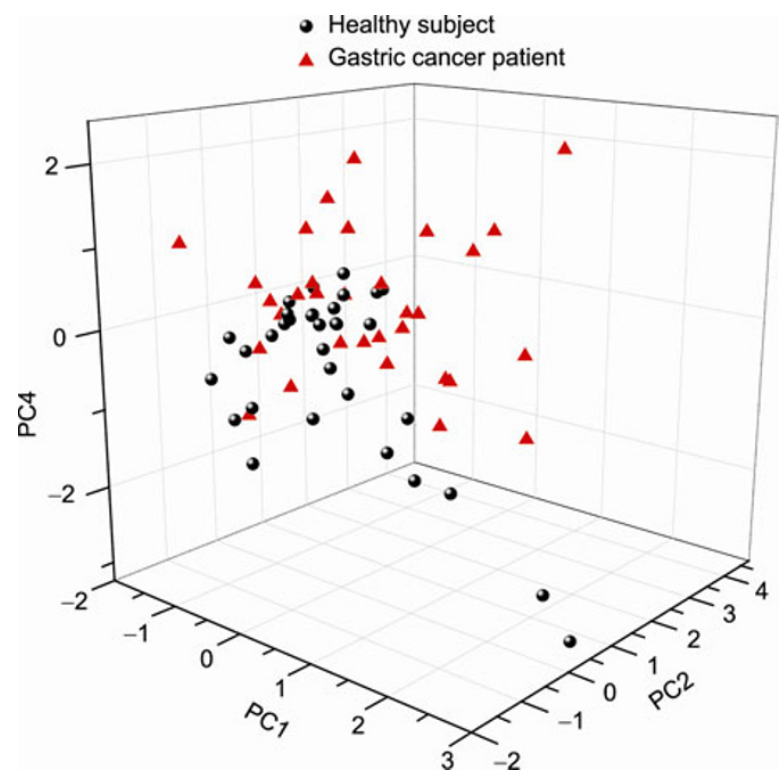

Figure 5 A three-dimensional mapping of the PCA result as calculated from the original SERS data for the gastric cancer group (red triangles) and the healthy volunteer group (black circles) with PC1, PC2 and PC4 as the three axes.

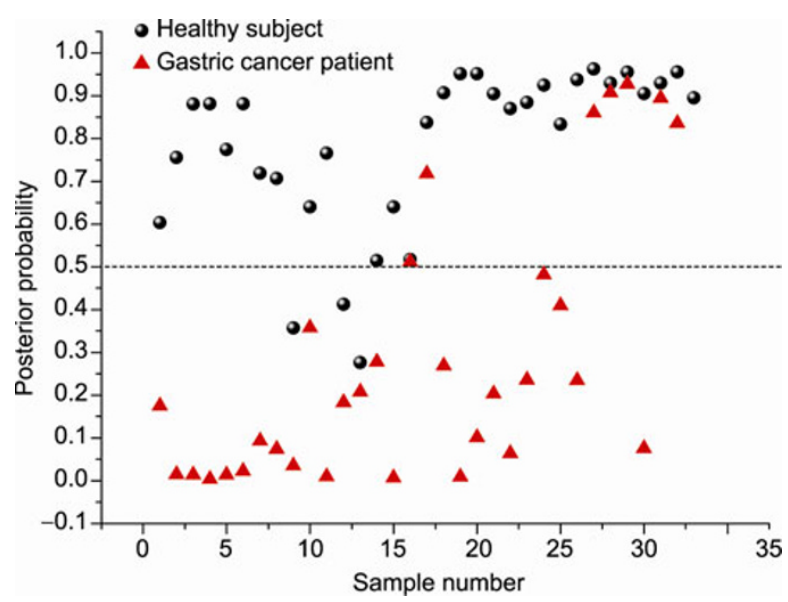

Figure 6 Scatter plots of the posterior probability of belonging to the normal (black circles) and gastric cancer (red triangles) categories using the PCA-LDA technique. The discrimination threshold was 0.5 . 
the normalization of the entire SERS spectrum data set, we found that the diagnostic specificity could be significantly increased. SERS data pretreatment can reduce adverse effects such as autofluorescence and experiment condition variations and facilitate more accurate spectral shape analysis to achieve a better diagnosis performance.

To further evaluate the performance of the PCA-LDAbased diagnostic algorithm for gastric cancer diagnosis, a receiver operating characteristic (ROC) curve was generated at different threshold levels (Figure 7). The data point labeled by the black arrow achieved a diagnostic sensitivity of $79.5 \%$ and specificity of $91 \%$, and the area under the ROC curve was 0.89 . The result shows that the blood plasma SERS spectra can be used for gastric cancer detection with high sensitivity and specificity.

\subsection{Discussion}

The results of our exploratory study demonstrate that there were specific differences in SERS spectra for blood plasma of gastric cancer patient versus blood plasma of healthy subjects, suggesting great potential for SERS in gastric cancer detection and screening applications. SERS spectra of blood plasma are dominated by many vibrational modes of various biomolecules, such as proteins, lipids and nucleic acids, which may change in quantity or conformation associated with neoplastic transformation. To better understand the molecular basis for the observed SERS spectra of human plasma, Table 1 lists tentative assignments for the observed SERS bands, according to data from the literature [33-39]. For example, the SERS peak at $1338 \mathrm{~cm}^{-1}$ due to the $\mathrm{C}-\mathrm{H}$ vibration of nucleic acid bases exhibited a higher signal in cancer plasma, indicating an increase in the percentage of the nucleic acid bases content relative to the total SERSactive components in the plasma of gastric cancer patients. This is in agreement with studies of esophageal cancer, breast cancer, liver cancer, lung cancer and prostate cancer [40].

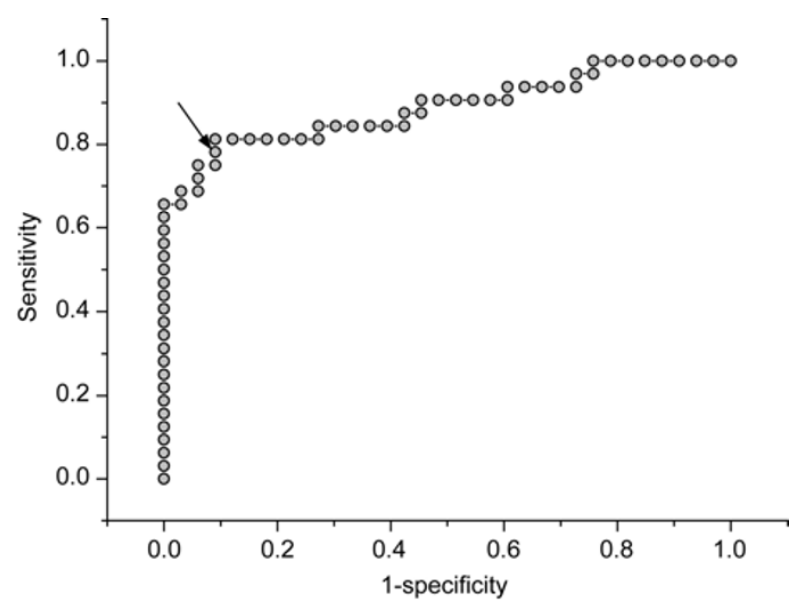

Figure 7 The receiver operating characteristic (ROC) curve of the discrimination result for the PCA-LDA-based SERS spectral classification.
Table 1 Peak positions and tentative assignments of major vibrational bands observed in plasma samples ${ }^{\text {a) }}$

\begin{tabular}{ccc}
\hline $\begin{array}{c}\text { Peak position } \\
\left(\mathrm{cm}^{-1}\right)\end{array}$ & Vibrational mode & Major assignments \\
\hline 494 & ring vibration & $\begin{array}{c}\text { cellulose, guanine, L-arginine } \\
\text { ascorbic acid, amide-VI }\end{array}$ \\
589 & $v(\mathrm{C}-\mathrm{S})$ & L-tyrosine, lactose \\
638 & $\delta(\mathrm{C}-\mathrm{H})$ & adenine, coenzyme A \\
725 & $v(\mathrm{C}-\mathrm{C}-\mathrm{O})$ & L-serine, glutathione \\
813 & $\delta(\mathrm{C}-\mathrm{O}-\mathrm{H})$ & glutathione, D-(C)-galactosamine \\
886 & $v(\mathrm{C}-\mathrm{C})$ & $\alpha$-helix, proline, valine \\
959 & $v s(\mathrm{C}-\mathrm{C})$ & phenylalanine \\
1004 & $v(\mathrm{C}-\mathrm{N})$ & collagen \\
1074 & $v(\mathrm{C}-\mathrm{N})$, & D-mannos \\
1095 & $v(\mathrm{C}-\mathrm{N})$, & D-mannos, \\
1134 & ring vibration & L-tryptophan, phenylalanine \\
1207 & $v(\mathrm{C}-\mathrm{H})$ & nucleic acid bases \\
1330 & $\delta(\mathrm{CH})$ & collagen, phospholipids \\
1445 & $\delta(\mathrm{C}=\mathrm{C})$ & phenylalanine, acetoacetate, ribo- \\
1580 & $v(\mathrm{C}=\mathrm{O})$ & flavin \\
1654 & $\alpha-h e l i x$, collagen \\
\hline
\end{tabular}

a) $v$, stretching vibration; $\delta$, bending vibration; vs, symmetric stretch.

Also, abnormal metabolism of DNA or RNA bases in the blood plasma of cancer patients may be detected in precancerosis. The reason for increased cell-free nucleic acid levels in cancer patients' blood remains largely unknown. The main mechanisms are proposed to be apoptosis and necrosis, or release of intact cells in the bloodstream [41]. The results show that the occurrence and development of cancer may be monitored by detecting the metabolism of DNA or RNA bases in the blood plasma of cancer patients.

The SERS band at $1445 \mathrm{~cm}^{-1}$ corresponds to the $\mathrm{C}-\mathrm{H}$ bending mode of collagen or phospholipids, and is higher in cancer plasma than in normal plasma. The peak has previously been recognized as being of diagnostic significance [35]. The SERS band at $1580 \mathrm{~cm}^{-1}$ was attributed to the $\mathrm{C}=\mathrm{C}$ bending mode of phenylalanine [37] and the percentage signals are considerably increased in cancer patient plasma, indicating an increase in the percentage of phenylalanine content relative to the total SERS-active components in the plasma of gastric cancer patients. Huang et al. also observed an increase of phenylalanine in malignant lung tissue by Raman spectroscopy [2]. The SERS bands of amide-VI $\left(589 \mathrm{~cm}^{-1}\right)$, L-tyrosine $\left(638 \mathrm{~cm}^{-1}\right)$, L-serine $(813$ $\left.\mathrm{cm}^{-1}\right)$, D-(C)-galactosamine $\left(886 \mathrm{~cm}^{-1}\right)$ and D-mannos $\left(1134 \mathrm{~cm}^{-1}\right)$ in plasma of gastric cancer patients show lower percentage signals than those of normal plasma, suggesting a decrease in the percentage of amino acids and saccharide contents relative to the total SERS-active components in plasma of gastric cancer patients. The reason for this may be the tumor's vigorous metabolism, which is consistent with other biochemical analysis results of tumor tissues.

PCA was used to define a new dimensional space in which the major variance in the original data set can be captured and represented by only a few principal component 
(PC) variables. These PCs are used to build a model with a resolution of recognition. Figure 4 shows the results of a three-dimensional scatter plot of PC scores (PC1, PC2 and PC7 as the three axes) for the healthy and cancer groups. We can clearly see that they were distributed in separate areas except for a small overlap. Figure 4 also shows that the regional distribution of the gastric cancer group is wider than that for the healthy control group. This is explainable. As the main medium for excretory product transportation, blood plasma delivers many dissolved nutrients, such as proteins, lipids, and sugars, to cells inside the entire body. Meanwhile, waste products are transported away from the same cells. Therefore, there are very complex components in blood plasma. For healthy people, the proportions of these components are relatively stable. However, under pathological conditions, cancer-related blood plasma constituents could be quite variable from patient to patient due to the heterogeneity of cancer development. These differences are probably responsible for the observed phenomenon that the regional distribution of the gastric cancer group on the PCA plot is much larger than for the healthy control group.

\section{Conclusion}

Silver nanoparticle-based, surface-enhanced Raman spectroscopy combined with multivariate analysis was applied to analyze the blood plasma from gastric cancer patients and healthy volunteers. Using a PCA-LDA diagnostic algorithm, we were able to differentiate gastric cancer from normal samples with high diagnostic sensitivity (79.5\%) and specificity $(91 \%)$. Tentative assignments of the Raman bands in the measured SERS spectra suggest interesting cancer-specific biomolecular differences, including an increase in the relative amounts of nucleic acid, collagen, phospholipids and phenylalanine and a decrease in the percentage of amino acids and saccharide contents in the blood plasma of cancer patients as compared with those of healthy subjects. These alterations may be due to the patients' body metabolic changes. The results from this exploratory study demonstrate great promise for developing SERS plasma analysis combined with multivariate analysis into a clinical tool for non-invasive detection and screening of gastric cancers.

This work was supported by the National Natural Science Foundation of China (Grant Nos. 60778046 and 60910106016), and the Project of Fujian Province (Grant Nos. 2009J01276 and 200810015).

1 Gremlich H U, Yan B. Infrared and Raman Spectroscopy of Biological Materials. New York: Marcel Dekker, 2000. 195-245

2 Huang Z, Mcwilliams A, Lui H, et al. Near-infrared Raman spectroscopy for optical diagnosis of lung cancer. Int J Cancer, 2003, 107: 1047-1052

3 Manoharan R, Shafer K, Perelman L, et al. Raman spectroscopy and fluorescence photon migration for breast cancer diagnosis and imaging. Photochem Photobiol, 1998, 67: 15-22
4 Shim M G, Wong L K S, Marcon N E, et al. In vivo near infrared Raman spectroscopy: demonstration of feasibility during clinical gastrointestinal endoscopy. Photochem Photobiol, 2000, 72: 146-150

5 Mahadevan-Jansen A, Mitchell M F, Ramanujam N, et al. Near infrared Raman spectroscopy for in vitro detection of cervical precancers. Photochem Photobiol, 1998, 68: 123-132

6 Stone N, Stavroulaki P, Kendall C, et al. Raman spectroscopy for early detection of laryngeal malignancy: preliminary results. Laryngoscope, 2000, 110: 1756-1763

7 Lau D, Huang Z, Lui H, et al. Raman spectroscopy for optical diagnosis in normal and cancerous tissue of the nasopharynx-preliminary findings. Laser Surg Med, 2003, 32: 210-214

8 Feng S, Lin J, Cheng M, et al. Gold nanoparticle based surface-enhanced Raman scattering spectroscopy of cancerous and normal nasopharyngeal tissues under near-infrared laser excitation. Appl Spectrosc, 2009, 63: 1089-1094

9 Tao J, Huang Y, Lin R, et al. A study on laser-Raman spectrometry for detecting signals of gastric cancerization (in Chinese). ACTA Laser Biol Sin, 2007, 16: 238-240

10 Leng A, Wang H, Yang J, et al. Application of laser resonance Raman spectroscopy in gastric cancer (in Chinese). China J Modern Med, 2009, 19: 2015-2019

11 Tao J, Huang Y, Lin R, et al. Differentiating gastric cancer cell from normal cell by laser Raman spectrum (in Chinese). Spectrosc Spect Anal, 2007, 27: 2262-2265

12 Tao Z, Yao H, Wang G, et al. Using Raman spectroscopy to analyze apoptosis of gastric cancer cells induced by cisplatin (in Chinese). Spectrosc Spect Anal, 2009, 29: 2442-2445

13 Zhang J, Shen A, Wei Y, et al. Study of normal mucosa and gastric carcinoma by confocal Raman microspectroscopy (in Chinese). J Biomed Engineer, 2004, 21: 910-912

14 Ling X, Li W, Song Y, et al. FT-Raman spectroscopic investigation on stomach cancer (in Chinese). Spectrosc Spect Anal, 2000, 20: 692-693

15 Tang W, Wang J, Xu P. Research of stomach cancer tissue by Raman spectroscopy (in Chinese). Laser J, 2004, 25: 82-83

16 Ellis D I, Goodacre R. Metabolic fingerprinting in disease diagnosis: biomedical applications of infrared and Raman spectroscopy. Analyst, 2006, 131: 875-885

17 Fleischman M, Hendra P J, McQuillan A J. Raman spectra of pyridine adsorbed at a silver electrode. Chem Phys Lett, 1974, 26: 163

18 Kneipp K, Kneipp H, Itzkan I, et al. Surface-enhanced Raman scattering: a new tool for biochemistry spectroscopy. Curr Sci, 1999, 77: 915

19 Zhang X, Young M A, Lyandres O, et al. Rapid detection of an anthrax biomarker by surface-enhanced Raman spectroscopy. J Am Chem Soc, 2005, 127: 4484

20 Zhang X, Zhao J, Whitney A V, et al. Ultrastable substrates for surface- enhanced Raman spectroscopy: A12O3 overlayers fabricated by atomic layer deposition yield improved anthrax biomarker detection. J Am Chem Soc, 2006, 128: 10304-10309

21 Bell S E J, Mackle J N, Sirimuthu N M S. Quantitative surface-enhanced Raman spectroscopy of dipicolinic acid-towards rapid anthrax endospore detection. Analyst, 2005, 130: 545-549

22 Taton T A, Mirkin C A. Scanometric DNA array detection with nanoparticle probes. Science, 2000, 289: 1757-1760

23 Cao Y W C, Jin R C, Mirkin C A. Nanoparticles with Raman spectroscopic fingerprints for DNA and RNA detection. Science, 2002, 297: $1536-1540$

24 Ji X, Xu S, Wang L, et al. Immunoassay using the probe-labeled $\mathrm{Au} / \mathrm{Ag}$ core-shell nanoparticles based on surface-enhanced Raman scattering. Colloid Surface A, 2005, 257-258: 171-175

25 Qian X, Peng X, Ansari D O, et al. In vivo tumor targeting and spectroscopic detection with surface-enhanced Raman nanoparticle tags. Nat Biotechnol, 2007, 26: 83-90

26 Ni J, Lipert R J, Dawson G B, et al. Immunoassay readout method using extrinsic Raman labels adsorbed on immunogold colloids. Anal Chem, 1999, 71: 4903-4908

27 Grubisha D S, Lipert R J, Park H Y, et al. Femtomolar detection of prostate-specific antigen: an immunoassay based on surface-en- 
hanced Raman scattering and immunogold labels. Anal Chem, 2003, 75: 5936-5943

28 Driskell J D, Kwarta K M, Lipert R J, et al. Low-lever detection of viral pathogens by a surface-enhanced Raman scattering based immunoassay. Anal Chem, 2005, 77: 6147-6154

29 Driskell J D, Uhlenkamp J M, Lipert R J, et al. Surface-enhanced Raman scattering immunoassays using a rotated capture substrate. Anal Chem, 2007, 79: 4141-4148

30 Feng S, Chen R, Lin J, et al. Nasopharyngeal cancer detection based on blood plasma surface-enhanced Raman spectroscopy and multivariate analysis. Biosens Bioelectron, 2010, 25: 2414-2419

31 Leopold N, Lendl B. A new method for fast preparation of highly surface-enhanced Raman scattering (SERS) active silver colloids at room temperature by reduction of silver nitrate with hydroxylamine hydrochloride. J Phys Chem B, 2003, 107: 5723-5727

32 Zhao J, Lui H, Mclean D I, et al. Automated autofluorescence background subtraction algorithm for biomedical Raman spectroscopy. Appl Spectrosc, 2007, 61: 1225-1232

33 Han H, Yan X, Dong R, et al. Analysis of serum from type II diabetes mellitus and diabetic complication using surface-enhanced Raman spectra (SERS). Appl Phys B, 2009, 94: 667-672

34 Uzunbajakava N, Lenferink A, Kraan Y, et al. Nonresonant Raman imaging of protein distribution in single human cells. Biopolymers,
2003, 72: 1-9

35 Liu C H, Das B B, Glassman W L, et al. Raman, fluorescence, and time-resolved light scattering as optical diagnostic techniques to separate diseased and normal biomedical media. Photochem Photobiol, 1992, 16: 187-209

36 Andrade P O, Bitar R A, Yassoyama K, et al. Study of normal colorectal tissue by FT-Raman spectroscopy. Anal Bioanal Chem, 2007, 387: $1643-1648$

37 Stone N, Stavroulaki P, Kendall C, et al. Raman spectroscopy for early detection of laryngeal malignancy: preliminary results. Laryngoscope, 2000, 110: 1756-1763

38 Gelder J D, Gussem K D, Vandenabeele P, et al. Reference database of Raman spectra of biological molecules. J Raman Spectrosc, 2007, 38: 1133-1147

39 Lyng F M, Faoláin E Ó, Conroy J, et al. Vibrational spectroscopy for cervical cancer pathology, from biochemical analysis to diagnostic tool. Exp Mol Pathol, 2007, 82: 121-129

40 Banki F, Yacoub W N, Hagen J A, et al. Plasma DNA is more reliable than carcinoembryonic antigen for diagnosis of recurrent esophageal cancer. J Am coll Surgeons, 2008, 37: 30-35

41 Gormally E, Caboux E, Vineis P, et al. Circulating free DNA in plasma or serum as biomarker of carcinogenesis: practical aspects and biological significance. Mutat Res-Rev Mutat, 2007, 635: 105-117

Open Access This article is distributed under the terms of the Creative Commons Attribution License which permits any use, distribution, and reproduction in any medium, provided the original author(s) and source are credited. 